\title{
Retraction
}

\section{Retraction: Winton et al., Intraneuronal APP, Not Free A $\beta$ Peptides in 3xTg-AD Mice: Implications for Tau Versus A $\boldsymbol{\beta}$-Mediated Alzheimer Neurodegeneration}

The Journal of Neuroscience has received notification of an investigation by the Perelman School of Medicine at the University of Pennsylvania, which supports the journal's findings of data misrepresentation in the article "Intraneuronal APP, Not Free A $\beta$ Peptides in 3xTg-AD Mice: Implications for Tau Versus A $\beta$-Mediated Alzheimer Neurodegeneration" by Matthew J. Winton, Edward B. Lee, Eveline Sun, Margaret M. Wong, Susan Leight, Bin Zhang, John Q. Trojanowski, and Virginia M.-Y. Lee, which appeared on pages 7691-7699 of the May 25, 2011 issue. Because the results cannot be considered reliable, the editors of The Journal are retracting the paper.

DOI:10.1523/JNEUROSCI.5236-14.2015 